\title{
Study on the stability of a shear-thinning suspension used in oil well drilling
}

\author{
Flávia M. Fagundes* , Nara B.C. Santos, João Jorge R. Damasceno, and Fábio O. Arouca \\ Federal University of Uberlândia, School of Chemical Engineering, Av. João Naves de Ávila, 2121, Bloco 1k, Uberlândia/MG, \\ 38.408-100 Santa Monica - Brazil
}

Received: 24 October 2017 / Accepted: 22 February 2018

\begin{abstract}
In order to avoid solid-liquid gravitational separation of particles in the drilling fluid and cuttings generated in this process, the oil industry has been developing drilling fluids with shear-thinning and thixotropic characteristics. In case of operational stops in the drilling process, the intense sedimentation of these particles can damage the equipment used and the well. In this context, this study simulated an operational stop to obtain information about stability of solids in a paraffin-based suspension with time-dependent shear-thinning behavior, which has already been used in current drilling processes. A long-term test using gamma-ray attenuation technique identified the separation dynamics of a set of micrometric particles belonging to and incorporated into the drilling fluid during operation. This test verified the typical regions of gravitational sedimentation and, through constant concentration curves, indicated that the sedimentation process did not occur at a constant rate. This study also proposed a constitutive equation for pressure on solids.
\end{abstract}

\section{List of notation}

$a, b, c, e$ Estimated parameters $\left[\mathrm{M}^{0} \mathrm{~L}^{0} \mathrm{~T}^{0}\right]$

$d \quad$ Estimated parameter $\left[\mathrm{M}^{-1} \mathrm{~L}^{-1} \mathrm{~T}^{-2}\right]$

$\beta \quad$ Calibration constant $\left[\mathrm{M}^{0} \mathrm{~L}^{0} \mathrm{~T}^{0}\right]$

$\varepsilon_{s} \quad$ Volumetric solids concentration $\left[\mathrm{M}^{0} \mathrm{~L}^{0} \mathrm{~T}^{0}\right]$

$\varepsilon_{s 0} \quad$ Initial volumetric solids concentration $\left[\mathrm{M}^{0} \mathrm{~L}^{0} \mathrm{~T}^{0}\right]$

$\varepsilon_{s m} \quad$ Maximum volumetric solids concentration $\left[\mathrm{M}^{0} \mathrm{~L}^{0} \mathrm{~T}^{0}\right]$

$g \quad$ Local gravity $\left[\mathrm{M}^{0} \mathrm{~L}^{1} \mathrm{~T}^{-2}\right]$

$I \quad$ Intensity of gamma-ray beams $\left[\mathrm{M}^{0} \mathrm{~L}^{0} \mathrm{~T}^{0}\right]$

$L \quad$ Sediment height $\left[\mathrm{M}^{0} \mathrm{~L}^{1} \mathrm{~T}^{0}\right]$

$m \quad$ Consistency index $\left[\mathrm{M}^{1} \mathrm{~L}^{-1} \mathrm{~T}^{-1}\right]$

$n \quad$ Behavior index $\left[\mathrm{M}^{0} \mathrm{~L}^{0} \mathrm{~T}^{0}\right]$

$\mu \quad$ Viscosity $\left[\mathrm{M}^{1} \mathrm{~L}^{-1} \mathrm{~T}^{-1}\right]$

$P_{s} \quad$ Pressure on solids $\left[\mathrm{M}^{1} \mathrm{~L}^{-1} \mathrm{~T}^{-2}\right]$

$\rho_{f} \quad$ Specific mass of fluid $\left[\mathrm{M}^{1} \mathrm{~L}^{-3} \mathrm{~T}^{0}\right]$

$\rho_{s} \quad$ Specific mass of solids $\left[\mathrm{M}^{1} \mathrm{~L}^{-3} \mathrm{~T}^{0}\right]$

$R \quad$ Corrected counting of radiation pulses $\left[\mathrm{M}^{0} \mathrm{~L}^{0} \mathrm{~T}^{-1}\right]$

$R_{0} \quad$ Corrected counting of radiation pulses crossing the test tube without solids $\left[\mathrm{M}^{0} \mathrm{~L}^{0} \mathrm{~T}^{-1}\right]$

$t \quad$ Time $\left[\mathrm{M}^{0} \mathrm{~L}^{0} \mathrm{~T}^{1}\right]$

$t_{m} \quad$ Resolution time of the system $\left[\mathrm{M}^{0} \mathrm{~L}^{0} \mathrm{~T}^{-1}\right]$

$\tau \quad$ Shear stress $\left[\mathrm{M}^{1} \mathrm{~L}^{-1} \mathrm{~T}^{-2}\right]$

$\gamma \quad$ Shear rate $\left[\mathrm{M}^{0} \mathrm{~L}^{0} \mathrm{~T}^{-1}\right]$

$z \quad$ Monitoring position $\left[\mathrm{M}^{0} \mathrm{~L}^{1} \mathrm{~T}^{0}\right]$

\section{Introduction}

In the drilling oil wells, different formulations of fluids have been used to present specific properties desirable in each stage of the process. The substances usually added are emulsifying, thickening and gelling agents that are pumped to the surface; lift the cuttings to the surface; cool, lubricate and reduce friction between machinery and the wellbore; apply a hydrostatic pressure on well walls to prevent the invasion of fluid in the reservoir rock [1-7].

Corrective or preventive operational stops interrupt the fluid flow used in drilling, and the solids concentration profile changes due to sedimentation of particles in the fluid and the cuttings generated in the operation. In this context, drilling fluids that can form gel structures in the absence of shear stress have been developed. Gelling prevents particle sedimentation and, consequently, damages caused by the accumulation of solids on the drill bit [7-9].

Although some studies have been carried out [10-17] on the behavior of settling particles in shear-thinning fluids, there is lack of information on this phenomenon in drilling fluids, especially in the compression zone.

Thus, the general aim of this study was to investigate solid-liquid separation in a fluid developed by an oil company, which has been already used in the field during an operational stop. We also intended to present a constitutive equation for pressure on solids. With these results and the equation of motion for solids, we aim to

\footnotetext{
* Corresponding author: flaviamfagundes@gmail.com
} 
reproduce in the future a real situation of inactive oil wells filled with the fluid studied.

\section{Experimental procedure}

\subsection{Fluid characterization}

The estimated parameters were: specific mass of solid $\left(\rho_{s}=2709 \mathrm{~kg} / \mathrm{m}^{3}\right)$ by helium pycnometer technique in the Micromeritics Gas Pycnometer, AccuPyc model 1330; specific mass of fluid $\left(\rho_{f}=1145.9 \mathrm{~kg} / \mathrm{m}^{3}\right)$ by simple pycnometry; and determination of initial solids concentration in suspension $\left(\varepsilon_{s 0} \approx 14 \%\right)$ by retort analysis using the FANN Model 210463 Kit with a $50 \mathrm{~mL}$ capacity.

\subsection{Solid characterization}

The solid content of suspension is formed by solids additives such as barite and cut solids from rock drilling.

The characterization of solids size was: $D_{0.1}=3.008 \mu \mathrm{m}$, $D_{0.5}=40.803 \mu \mathrm{m}$ and $D_{0.9}=232.247 \mu \mathrm{m}$ (laser granulometer, Malvern Mastersizer MicroPlus MAF $5001^{\circledR}$ ).

\subsection{Rheology}

To characterize the rheological properties of fluid, the hysteresis and flow curve were determined. We performed tests in triplicate and with new samples, at $25^{\circ} \mathrm{C}$ in the Brookfield model $\mathrm{R} / \mathrm{S}$ Plus rheometer, geometrically in rotary cone and fixed plate, with a Brookfield thermostatic bath, programmable controller model TC-6021.

Samples were submitted to intense pre-shear (rate of $1050 \mathrm{~s}^{-1}$ ) for $1 \mathrm{~min}$. To assess viscosity dependence over time, we conducted tests varying the strain rates from $1 \mathrm{~s}^{-1}$ to $1050 \mathrm{~s}^{-1}$. Then, we analyzed the area of hysteresis between the ascending curves followed by a decrease in the strain rate. To construct the flow curve, we submitted the samples to constant shear rates of $200,400,600,800$, and $1000 \mathrm{~s}^{-1}$ until they reached the steady state, when the shear stress values were obtained and adjusted to the Power-law model (Eq. 1).

$$
\tau=m \gamma^{n},
$$

where $m$ and $n$ are indices of consistency and behavior, respectively, $\gamma$ is the shear stress, and $\gamma$ is the shear rate.

\subsection{Gamma-ray attenuation technique}

Gamma-ray attenuation measurements is a technique used to obtain indirect volumetric concentration of solids in batch sedimentation tests without interfering in the configuration and stability of the medium. This technique was applied in studies of gravitational sedimentation in Newtonian fluids [18-20] and laboratory produced shearthinning fluids [16].

The radioisotope application unit, represented by the flow chart of Figure 1, consisted of a radiation targeting/ detection system coupled to a metal structure. This metal structure had a mobile platform that allowed the horizontal beam of radiation to be positioned at different heights, allowing monitoring of concentration from the

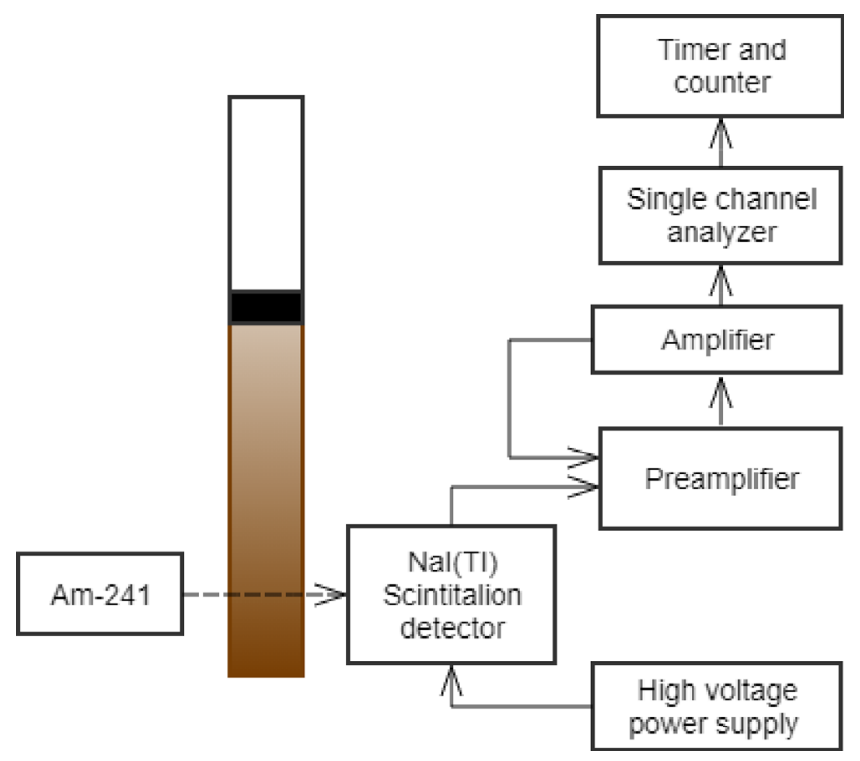

Fig. 1. Illustration of the radioisotope application system.

bottom of the test vessel $(z=0 \mathrm{~cm})$ to the top of the suspension column $(z=21 \mathrm{~cm})$. We placed the homogenized fluid in a glass test tube with the following dimensions: $350-\mathrm{mm}$ high, $55-\mathrm{mm}$ internal diameter, and 3 -mm thick. We positioned the glass tube in the center of the radioisotope application unit.

In order to guarantee that the experimental unit operated in optimal condition, we used pre-scaled results [19]: the high voltage source was $900 \mathrm{~V}$, and the radioisotope energy range was $500-800 \mathrm{mV}$ (Americium-241).

To obtain local solids concentration by means of gamma-ray attenuation technique, we previously corrected the intensity of gamma-ray beam $(I)$ with the system's resolution time $\left(t_{m}=240 \pm 50 \mu \mathrm{s}\right)$ (Eq. 2). Then, we used Lambert's equation [21], (Eq. 3):

$$
\begin{gathered}
R=\frac{I}{1-t_{m} I}, \\
\ln \left(\frac{R_{0}}{R}\right)=\beta \varepsilon_{s},
\end{gathered}
$$

where $R$ and $R_{0}$ represent the corrected counting of pulses arriving at the detection system after passing through a solution with and without suspended solids, respectively; $\varepsilon_{s}$ is the volumetric concentration of solids; and $\beta$ is a calibration constant.

To determine $\beta$, we used the pulse counting when the suspension was homogenized, which corresponded to the initial volumetric solids concentration $\left(\varepsilon_{s 0}\right)$, and the pulse counting in the region of zero solids concentration. The value of the calibration constant was 0.205 .

\subsection{Constant concentration curves}

We organized the volumetric solids concentration values obtained through gamma-ray attenuation technique with the time of appearance in each position monitored, which allowed the construction of constant concentration curves. 


\subsection{Pressure on solids}

Determining the constitutive law of a fluid is a characterization very important to maintain the process security [6]. The determination of a constitutive equation for pressure on solids $\left(P_{s}\right)$ started with an adjustment of the values of volumetric solids concentration in the sediment as a function of the monitoring position, according to Equation (4) [20].

$$
\varepsilon_{s}=\frac{c+a z}{1+b z}
$$

where $a, b$ and $c$ are estimated parameters.

We also considered the flow through the unidimensional, permanent, slow porous medium and tension on solids as a function of the local porosity. We did not consider the inertial terms of the equation of motion for solids [20].

Thus, assuming that the medium was static, we used the following expression for pressure on solids [20], (Eq. 5):

$$
P_{s}=\left(\rho_{s}-\rho_{f}\right) g \int_{0}^{L} \varepsilon_{s} d z
$$

where $z$ represents the reference axis measured from the top of the sediment height $L, \rho_{s}$ and $\rho_{f}$ are the specific mass of solids and fluid, respectively, and $g$ is the local gravity.

Finally, Equation (6) related the volumetric concentration with pressure on solids.

$$
P_{s}=d \varepsilon_{s}^{e},
$$

where $d$ and $e$ are estimated parameters.

\section{Results}

\subsection{Rheological analysis}

We confirmed the fluid pseudoplasticity through the flow curve and by fitting the Power-law model to the experimental results, as shown in the log-log ladder, in Figure 2.

Table 1 shows indices of consistency and behavior of the rheological model and the determination coefficient, $r^{2}$.

The index of behavior presented a value lower than 1 , confirming the shear-thinning behavior of the drilling fluid studied $[5,22,23]$.

We determined the time dependence by the hysteresis formed (Fig. 3) and by the time required (approximately $3.5 \mathrm{~h}$ ) for the fluid to reach the steady state in the tests with constant shear rate [22, 23].

\subsection{Analysis of volumetric solids concentration profile}

A $500 \mathrm{~mL}$ suspension sample $(21 \mathrm{~cm}$ high in the test vessel) was monitored by the gamma-ray attenuation technique for the period of one year. Figure 4 shows the monitoring results for all positions from the bottom of the glassware.

When analyzing the curves from $z=0.5 \mathrm{~cm}$ to $z=12 \mathrm{~cm}$, the increase in solids concentration was slow. It was attributed to the rheological propriety of suspension. As the suspension showed shear-thinning and thixotropic

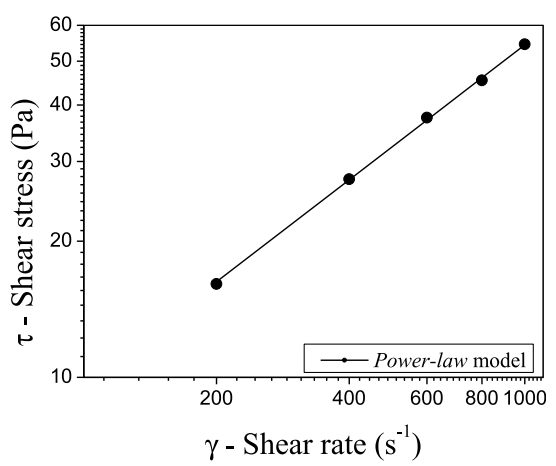

Fig. 2. Rheological curve.

Table 1. Parameters and determination coefficient of Power-law model.

\begin{tabular}{lll}
\hline \multicolumn{2}{c}{ Parameters } & Determination coefficient \\
\hline$m$ & $n$ & $r^{2}$ \\
0.307 & 0.749 & 0.9997 \\
\hline
\end{tabular}

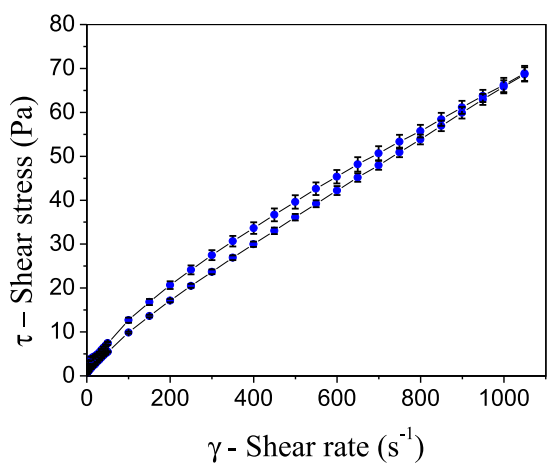

Fig. 3. Hysteresis loop area.

behavior, as the time goes on the polymers structures presented in the fluid had become gelified, which increased the polymeric arrangement of sediment column structure to be passed through by solids and form the sediment. The same was verified in another study with drilling fluids [24].

In this context, the deformation caused by shear rate sedimentation was very low and the agglomeration phenomenon also from solid particles sedimentation in the sediment structure was not observed. So, although the suspension had non-Newtonian nature, the sediment profile present Newtonian characteristics, and the velocity of sedimentation was guide by fluid flow upward against gravitational solid deposition to the bottom tube [14, 24].

When evaluating the sedimentation process, the effect of concentration should be considered. The set of particles during sedimentation (downwards) pushes the fluid in the lower positions so it only has one possible way, goes vertically upwards and therefore decelerate the sedimentation. This phenomenon was observed in Newtonian fluids $[16,14]$. 


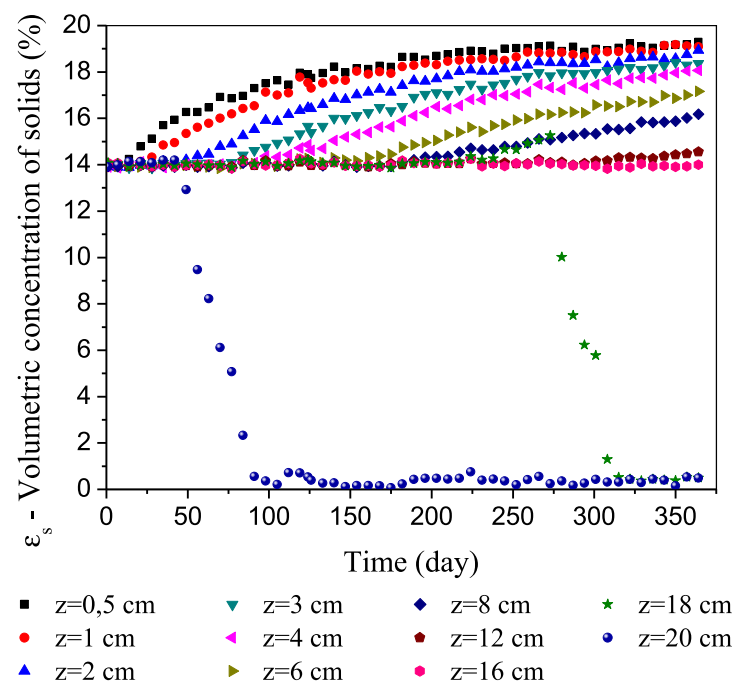

Fig. 4. Variation in solids concentration as a function of time.

When monitoring from $z=0.5$ to $16 \mathrm{~cm}$, the following sedimentation regions were observed:

- from the beginning of the experiment up to approximately 20 days, the suspension was homogenized and, therefore, in the region of free sedimentation $\left(\varepsilon_{s}=\varepsilon_{s 0}\right)$;

- in the monitoring performed from days 21 to 249 , two regions were found: the intermediate one, in which the concentration of particles was between the maximum (sediment concentration) and the initial concentration of the suspension $\left(\varepsilon_{s m}<\varepsilon_{s}<\varepsilon_{s 0}\right)$, and the region of free sedimentation;

- from days 250 to 364 , positions closest to the bottom of the test vessel $(z=0.5 \mathrm{~cm}$ and $z=1 \mathrm{~cm})$ had the highest volumetric particle concentration values $\left(\varepsilon_{s m} \cong 19 \%\right)$ and tended to stability. We also observed the intermediate regions $(z=2 \mathrm{~cm}$ to $z=12 \mathrm{~cm})$ and free sedimentation $(z=16 \mathrm{~cm})$.

The inclination of the curves decreased with the increase in the monitoring position; therefore, we assumed that, initially, larger particles reached the bottom of the vessel, and the smaller particles, after a certain time, filled the interstices, increasing sediment concentration until the final stability condition $[16,19,25,26]$.

Figure 5 shows the results of monitoring the local solids concentration over time for positions $z=18$ and $20 \mathrm{~cm}$.

Figure 5 shows that solids concentration remained constant for a period and then decreased to values close to zero. Such behavior was due to the passage of superior discontinuity through the radiation detection system.

In addition, for position $z=18 \mathrm{~cm}$, monitoring showed a peak of solids concentration during $9.6 \%$ of the experiment time. According to theory [27], this behavior is related to the increase in the intermediate region before the solids concentration tend to zero.

The concentration reduction at positions near the top of the fluid occurred linearly and slowly. For position $z=20 \mathrm{~cm}$, the time required to reduce the initial concentration to close to zero was approximately 90 days, and, for position $z=18 \mathrm{~cm}$, this time was approximately 315 days. Figure 6 clearly shows the region with solids concentration

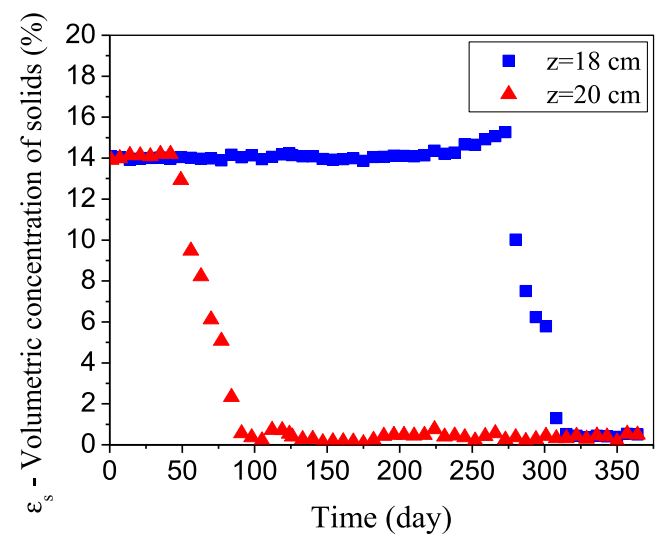

Fig. 5. Variation in solids concentration as a function of time to the $18 \mathrm{~cm}$ and $20 \mathrm{~cm}$ positions.

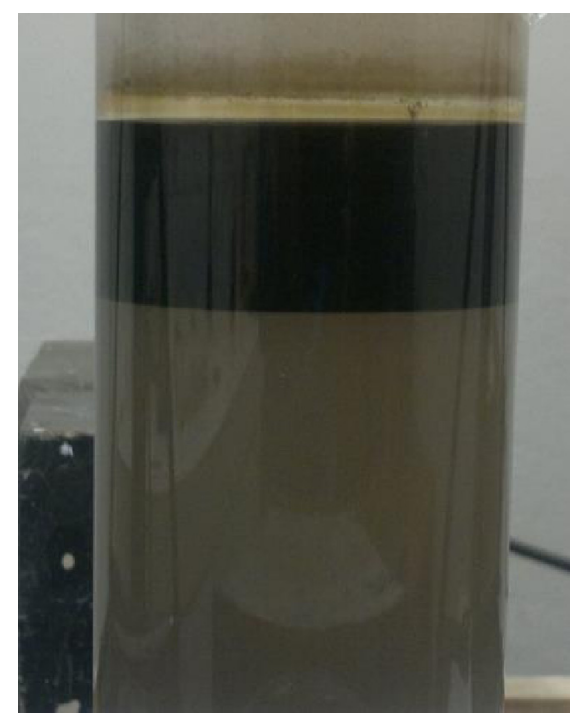

Fig. 6. Region with local solids concentration close to zero.

close to zero, due to the different coloring of the fluid. Although the literature [27] has named this region as clarified liquid, the fluid studied had a dark coloring.

The deviations obtained for each monitoring ranged from $0.1864 \%$ to $0.0640 \%$.

The relationship between position and time in which curves had the same concentration value, the constant concentration curves, allowed to interpret sedimentation as the propagation of waves with equal concentration and to evaluate the characteristics of the materials settling in the drilling fluid [28] (Fig. 7).

Figure 7 shows that the constant concentration curves associated with sediment formation presented different inclinations. The concentration curves closer to the initial volumetric solids concentration in the suspension were the most inclined. Therefore, sedimentation did not occur at a constant rate.

We also observed the relationship between the time of appearance of constant concentration curves as a function of concentration, i.e., the curves representing higher concentrations took longer to emerge. Thus, constant 


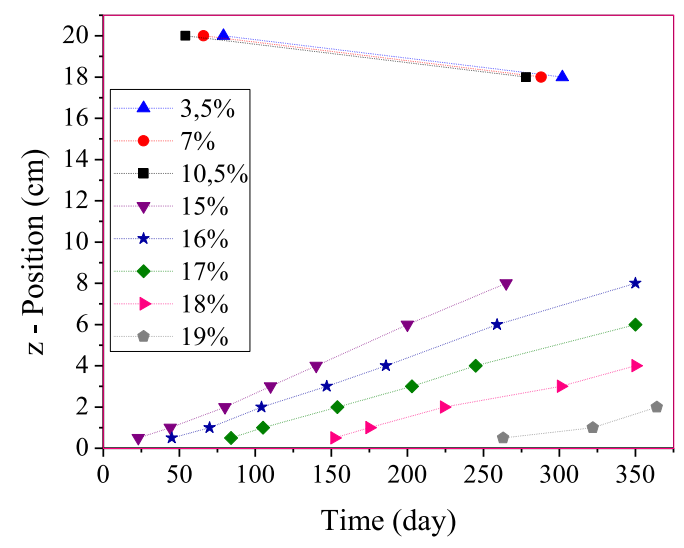

Fig. 7. Constant concentration curves.

Table 2. Parameters for the adjustment of concentration versus position.

\begin{tabular}{ccc}
\hline$a$ & $b$ & $c$ \\
\hline 0.015 & 0.049 & 0.135 \\
\hline
\end{tabular}

concentration curves are not straight and do not start in the origin of the axes. This behavior is related to the compression of sediment caused by the upper layers of solids [19, 29].

Pressure on solids was another parameter. We adjusted the results of volumetric solids concentration in the sediment formed after one year of experiment as a function of the position (Eq. 4). The determination coefficient for fitting was 0.997. Table 2 presents the parameters determined.

The adjustment calculated pressure on solids. Subsequently, we adjusted the values calculated by Equation (5) to calculate pressure on solids as a function of concentration, according to Figure 8 and Table 3.

\section{Conclusion}

Monitoring solids concentration over time allowed a quantitative and qualitative evaluation of the sedimentation of particles in drilling fluids. The suspension presented good stability when compared to others that also have time-dependent shear-thinning characteristics.

The results indicated the trend of logarithmic growth of the curves of volumetric solids concentration versus time for the monitoring performed near the bottom of the test vessel. For positions near the top, this behavior was linear.

Constant concentration curves enabled the verification of compressibility of the sediment formed and the occurrence of different settling rates.

This study proposed a constitutive equation for pressure on solids, assuming that the system was static.

Acknowledgments. The authors thank Capes, CNPq, Fapemig, Petrobras, and the School of Chemical Engineering of the Federal University of Uberlandia for the financial support.

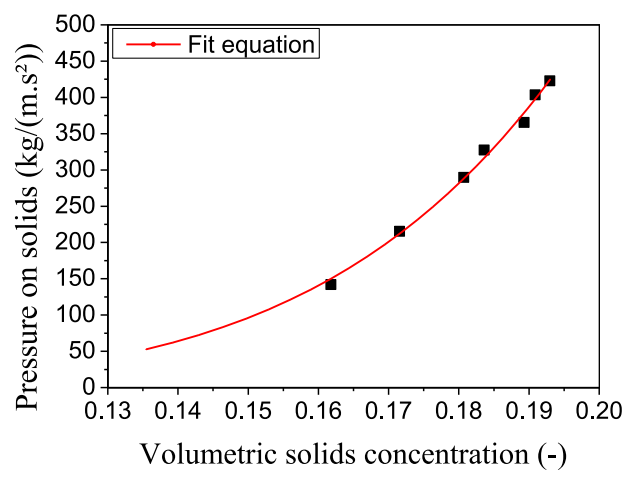

Fig. 8. Pressure on solids as a function of sediment concentration.

Table 3. Parameters of the adjustment performed for pressure on solids as function of volumetric solids concentration and determination coefficient.

\begin{tabular}{lcc}
\hline$d\left(1 /\left(\mathrm{kg} \mathrm{m} \mathrm{s}^{2}\right)\right)$ & $e$ & $r^{2}$ \\
\hline 6863573 & 5.891 & 0.986 \\
\hline
\end{tabular}

\section{References}

1 Thomas J.E. (2001) Fundamentos de engenharia de petróleo, Interciência, Rio de Janeiro.

2 Caenn R., Darley H.C.H., George R.G. (2011) Composition and properties of drilling and completion fluids, Gulf Professional Publishing, Oxford.

3 Peysson Y. (2004) Solid/Liquid Dispersions in Drilling and Production, Oil Gas Sci. Technol., 59, 1, 11-21.

4 Ghalambor A., Ashrafizadeh S.N., Nasiri M.Z. (2008) Effect of basic parameters on viscosity in synthetic-based drilling fluids, Soc. Pet. Eng. DOI: 10.2118/112276-MS

5 Henaut I., Pasquier D., Rovinetti S., Espagne B. (2015) HPHT Drilling mud based on environmently-friendly fluorinated chemicals, Oil Gas Sci. Technol. 70, 6, 917-930.

6 Van Damme H. (2004) Éditorial, Oil Gas Sci. Technol. 59, 1, 7-10.

7 Coussot P., Bertrand F., Herzhaft B. (2004) Rheological behavior of drilling muds, characterization using MRI visualization, Oil Gas Sci. Technol. 59, 1, 23-29.

8 Gandelman R.A., Pinto G.H.V.P. (2009) Desenvolvimento de metodologia e correlações para previsão de perfil de concentração de sólidos durante a perfuração de poços de petróleo em períodos de estática, Boletim técnico de produção de petróleo, Rio J. 4, 2, 261-273.

9 Oliveira G.M., Rocha L.L.V., Franco A.T., Negrâo C.O.R., Martins A.L. (2007) Reinício da circulação de fluidos de perfuração gelificados, $4^{\circ}$ Congresso Brasileiro de P\&D em Petróleo de Gás, 2. 2.0113-1-1.

10 Allen E., Uhlherr P.H.T. (1989) Nonhomogeneous sedimentation in viscoelastic fluids, J. Rheol. 33, 627-638.

11 Bobroff S., Phillips R.J. (1998) Nuclear magnetic resonance imaging investigation of sedimentation of concentrated suspensions in non-newtonian fluids, J. Rheol. 42, 1419-1436.

12 Daugan S., Talini L., Herzhaft B., Allain C. (2002) Aggregation of particles settling in shear-thinning fluids. Part 1. Two-particle aggregation, Eur. Phys. J. E 7, 73-81. 
13 Daugan S., Talini L., Herzhaft B., Allain C. (2002) Aggregation of particles settling in shear thinning fluids. Part 2. Three particle aggregation, Eur. Phys. J. E 9, 5562.

14 Daugan S., Talini L., Herzhaft B., Peysson Y., Allain C. (2004) Sedimentation of suspensions in shear-thinning fluids, Oil Gas Sci. Technol. 59, 71-80.

15 Gueslin B., Talini L., Herzhaft B., Peysson Y., Allain C. (2006) Aggregation behavior of two spheres falling through an aging fluid, Phys. Rev. 74, 042501.

16 Moreira B.A., Arouca F.O., Damasceno J.J.R. (2017) Analysis of suspension sedimentation in fluids with rheological shear-thinning properties and thixotropic effects, Powder Technol. 308, 290-297.

17 Ribeiro J.M., Eler F.M., Martins A.L., Scheid C.M., Calçada L.A., Meleiro L.A.C. (2017) A simplified model applied to the barite sag and fluid flow in drilling muds: Simulation and experimental results, Oil Gas Sci. Technol. 72, 23.

18 Damasceno J.J.R. (1992) Uma Contribuição ao Estudo do Espessamento Contínuo, Doctoral Thesis, Federal University of Rio de Janeiro, Rio de Janeiro, 164 p.

19 Arouca F.O. (2007) Uma Contribuição ao Estudo da Sedimentação Gravitacional em Batelada, Doctoral Thesis, Federal University of Uberlândia, Uberlândia, 286 p.
20 Arouca F.O., Damasceno J.J.R. (2005) The use of high energies radiations to characterise solid-liquid systems, Mater. Sci. Forum 498, 49-54.

21 Gardner R.P., Ely-Jr R.L. (1967) Radioisotope measurement applications in engineering, Reinhold Publ, New York.

22 Chhabra R.P., Richardson J.F. (2008) Non-newtonian flow and applied rheology, Butterworth Heinemann, Oxford.

23 Machado J.C.V. (2002) Reologia e escoamento de fluidos Ênfase da indústria do petróleo, Interciência, Rio de Janeiro.

24 Santos N.B.C., Fagundes F.M., Arouca F.A., Damasceno J.J. R. (2018) Sedimentation of solids in drilling fluids used in oil well drilling operations, J. Pet. Sci. Eng. 162, 137-142.

25 Comings E.W. (1940) Thickening calcium carbonate slurries, Ind. Eng. Chem. 32, 5, 663-667.

26 Work L.T., Kohler A.S. (1940) Sedimentation of suspensions, Ind. Eng. Chem. 32, 10, 1329-1334.

27 Kynch G.J. (1952) A theory of sedimentation, Trans. Amer. Soc. 48, 166-176.

28 Arouca, F.O., Lopes, L.C.O., Damasceno, J.J.R. (2006) Concentration profiles and iso-concentration curves for batch settling using the gamma rays attenuation technique, Mater. Sci. Forum 530, 29-34.

29 Concha F., Bustos M.C. (1987) Modification of the kynch theory of sedimentation, AIChE J. 33, 2, 312-315. 